\title{
Computational Intelligence in Astronomy
}

\author{
Ping Guo ${ }^{1}$, Yuping Wang ${ }^{2}$, Hailin $\mathrm{Liu}^{3}$, and Yiu-ming Cheung ${ }^{4}$ \\ ${ }^{1}$ School of Systems Science, Beijing Normal University, Beijing 100875, China \\ E-mail:pguo@bnu.edu.cn \\ ${ }^{2}$ School of Computer Science and Technology, Xidian University, Xian 710071, China \\ E-mail: ywang@xidian.edu.cn \\ ${ }^{3}$ School of Applied Mathematics, Guangdong University of Technology, Guangzhou 510520, China \\ E-mail: hlliu@gdut.edu.cn \\ ${ }^{4}$ Department of Computer Science, Hong Kong Baptist University, Hong Kong SAR, China \\ E-mail: ymc@comp.hkbu.edu.hk
}

\section{Preface}

The computational intelligence (CI) is the new stage of artificial intelligence (AI) developments. It is believed that $\mathrm{CI}$ is reinventing the study of modern astronomy. Accordingly, we aim to address the recent theoretical and practical developments, as well as the empirical studies, of CI in astronomy. This special issue is mainly dedicated to the workshop of AI in astronomy, in conjunction with the 12th International Conference on Computational Intelligence and Security (CIS 2016) held in Wuxi, China on December 16-19, 2016, which has 136 final accepted papers selected from 288 online submissions. Parts of submission of this special issue are through open Call-for-Papers posted in the web site of IJCIS. After peer review, 4 revised and extended papers are finally accepted, which reflect the current development of CI in astronomy.

The first paper by Ke Wang, Ping Guo, Fusheng Yu, Lingzi Duan, Yuping Wang, and Hui Du is entitled "Computational Intelligence in Astronomy: A Survey". The authors gave a review for the current state of the application of computational intelligence in astronomy. They believed that computational intelligence can provide powerful tools for addressing challenges in astronomical data analysis. With this emerging crossdisciplinary studies, it is expected that their work could establish collaborative relationships with experts in astronomy and CI, and help to promote the development of both $\mathrm{AI}$ and astronomy research fields.

The second paper by Xiaoyan Cai, Junwei Han, Shirui Pan, and Libin Yang is entitled "Heterogeneous Information Network Embedding based Personalized Query-Focused Astronomy Reference Paper Recommendation". The authors studied the problem of personalized query-focused astronomy reference paper recommendation and proposed a heterogeneous information network embedding based recommendation approach. The effectiveness of they proposed method has been demonstrated by the recommendation evaluation conducted on the IOP astronomy journal database.

The third paper by Xiaoli Wang, Bharadwaj Veeravalli, and Omer F. Rana is entitled "An Optimal Task-Scheduling Strategy for Large-Scale Astronomical Workloads using In-transit Computation Model'. The authors developed an optimal task-scheduling strategy by using in-transit computation model under fog 
computing to improve the processing efficiency of the large-scale astronomical data. They conducted various experiments to illustrate the correctness and effectiveness of the proposed strategy. Experimental results show that it can significantly decrease the processing time of large-scale workloads.

The fourth paper by J.P. Córdova Barbosa, S.G. Navarro Jiménez, J.C. Ramírez Vélez is entitled "Machine Learning for Stellar Magnetic Field Determination". The authors presented the results for the automatic determination of the mean longitudinal magnetic field in polarized stellar spectra through the analysis of spectropolarimetric observations. They developed a synthetic database encompassing a set of different stellar spectra, each of which is defined by a set of free parameters. They used supervised learning for artificial neural networks technique to achieve the goal of stellar magnetic field automatic determination.

Finally, the Guest Editors wish to express our deep appreciation to the authors for their contribution, to the reviewers for their careful, insightful and constructive reviews that led to further improvement of the articles. We are thankful to Prof. Luis Martinez and Prof. Jie $\mathrm{Lu}$, Editors-in-Chief of the Journal, for accepting to publish this Special Issue and for their help throughout the publication process. 